\title{
Iconographie des forces de l'ordre dans Les gardiens du temple de Cherkh Hamidou Kane
}

\section{Babacar NDIAYE}

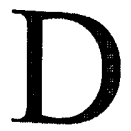

ans la thématique et la classification' de la littérature africaine post-coloniale, la figure de l'autorité, principalement représentée par le personnage du dictateur, a tenu une bonne place. L'exercice ainsi que le contrôle effectif du pouvoir se sont généralement faits par ou avec le soutien complice de l'appareil militaire. L'autorité apparaissait le plus souvent dans un uniforme, symbolisant son appartenance à cette entité de l'Etat, et tous ses faits et gestes s'accompagnaient d'un cérémonial martial très solennel caractéristique du militaire. Evidemment, cela correspondait à une certaine réalité politique africaine dans laquelle les romanciers s'abreuvaient avant de la restituer sous une forme imagée, caricaturale et parfois profondément satirisée. ${ }^{2}$

C'est pourquoi d'ailleurs Les gardiens du temple constitue une œuvre marquée du sceau de la diférance, et qu'il convient de qualifier comme une production littéraire décontextualisée. ${ }^{3}$ L'auteur s'inspire manifestement de faits réels survenus dans son pays, événements vécus par lui avec une proximité affective certaine, et dont les conséquences n'ont pas manqué d'impact dans son parcours professionnel en tant que fonctionnaire et autorité ministérielle par la suite. Mais le texte a été publié tardivement, bien après les œ uvres de la même classe thématique de la période concernée, et surtout avec des effets optiques propres à l'écriture romanesque. ${ }^{4}$ 
Toujours est-il que ce roman de Cheikh Hamidou Kane exprime une certaine réalité bien africaine propre à la sphère du pouvoir, se manifestant par des crises et des luttes intenses face à un contre-pouvoir omniprésent. La dialectique pouvoir/contre-pouvoir offre généralement une vision manichéenne des personnages qui appartiennent plus ou moins à l'un ou l'autre côté comme les deux faces de Janus qu'une ligne imaginaire partage et différencie fondamentalement.

Dans le procès des indépendances et des régimes issus de cette mutation historique, les romanciers s'appuyaient sur cette perspective pour mieux critiquer les formes d'oppression, dans un dessein de libération à forte dose humaniste. En effet, selon Jacques Chevrier, l'image désespérée de l'Afrique de cette époque est que “. . . le pouvoir s'y manifeste sous les formes d'un totalitarisme appuyé à la fois sur la force militaire, la bureaucratie et l'idéologie."'s

Paradoxalement, Cheikh Hamidou Kane innove dans cet espace thématique dans la mesure où il fait apparaitre un autre rôle, et subvertit en partie le jeu de l'une des catégories conflictuelles pour enfin créer une tierce partie. Cette dernière se présente d'ailleurs comme un arbitre qui participe au jeu et dont la présence est une condition sine qua non de son déroulement et de son aboutissement. Ainsi, les forces de l'ordre apparaissent sous un autre jour pour donner une nouvelle image d'elles. Elles s'interposent, entre le pouvoir et le contre-pouvoir, symbolisant ainsi une ligne de démarcation constitutive de la physionomie de la scène politique et sociale. De ce fait, ces forces se donnent une identité, un caractère, une personnalité et une fonctionnalité spécifiques dans cette confrontation entre l'autorité politique et la population ou les organisations sociales, lesquelles développent des modalités de refus et de résistance en faisant valoir des revendications politiques, économiques, corporatives...

En fait, les rapports dialectiques entre le pouvoir et le contre-pouvoir permettent de mettre en évidence le rôle que jouent les forces, en principe neutres, dans la mesure où elles 'appliquent' la loi contre les abus des uns et contre les ambitions démesurées des autres, pour la sauvegarde du contrat de société. C'est pourquoi, sans nous aventurer dans la voie des techniques du récit, nous prenons ici l'option d'une étude des personnages dans une dynamique qui les situe dans la sphère des affrontements, des tensions et des crises que gère le pouvoir politique face au contre-pouvoir. Les différentes forces qui interviennent dans ce champ ont chacune leurs spécificités, leur rôle particulier dans le dispositif du maintien de l'ordre, et leurs rapports particuliers au public et au pouvoir. 
Ainsi, nous en arrivons à souligner la place importante de protagonistes non décisifs, mais utiles, certes, dans la problématique de l'ordre public au regard des libertés collectives, à travers le scénario mis en œ uvre par Cheikh Hamidou Kane.

\section{I - Le cadre général de l'action et les modalités des troubles publics}

Le titre de l'ouvrage semble très évocateur. En effet, de prime abord il fait penser à de vaillants soldats veillant jalousement sur un sanctuaire ou un territoire (en réalité Farba Mari et Daba Mbaye sont les incarnations vivantes des gardiens du temple ${ }^{6}$ ). Mais, par-delà la conservation des traditions séculaires face aux effets dévastateurs de la colonisation, l'omniprésence du désordre public traduit de facto la nécessité d'une régulation sociale, ce dont l'administration a la responsabilité. Celle-ci s'exécute par le biais des forces de l'ordre. La réunion présidée par le directeur de cabinet du ministre de l'intérieur en dit long ${ }^{7}$, mais surtout les propos sans ambiguïté du colonel Dimbo de l'état-major: "Il semble que l'ordre est la priorité numéro un dans les besoins de nos pays, sans quoi non seulement rien ne sera construit, mais encore nous nous ridiculiserons aux yeux du monde" (220).

En réalité, la problématique de l'ordre public traverse le roman de part en part. Il y a toujours un fait ou événement qui crée, favorise ou amplifie les rassemblements dans les espaces publics, avec implication ou intervention possible des forces de l'ordre. Ce sont ces mêmes événements qui permettent aux policiers, gendarmes, et soldats de l'armée d'être au contact des populations rassemblées ou en face des manifestants, de se produire dans leur rôle tampon entre ces derniers et le pouvoir, et aussi à leurs grands chefs hiérarchiques d'évoluer dans les hautes sphères politiques de décision par rapport à ces dérèglements sociaux trouvant leur apothéose dans la rue. Et à côté de ces manifestations des libertés collectives, il y a également les réunions que l'on pourrait qualifier de privées (conférence de Daba Mbaye, réunion du bureau de l'Intersyndicale). Toutes ces rencontres scandent le roman de Cheikh Hamidou Kane et constituent les principaux piliers de sa trame. Le roman se déroule sous la forme d'une consécution de rencontres, d'événements, de réunions, de rassemblements et d'attroupements sur la voie publique, avec des motifs, des allures et des incidents variés, en étroite relation avec la notion d'ordre, impliquant par là-même l'intervention des forces de sécurité et l'attention des autorités publiques. 
Concrètement, les populations des différents espaces décrits par le roman sont préoccupées par l'exercice complet de leurs libertés collectives et publiques. Qu'elles s'inscrivent ou non dans le cadre de la légalité, sans quoi cet attribut essentiel des communautés humaines se viderait de son sens, ces populations entendent jouir pleinement de leurs libertés collectives, aussi bien en milieu rural que dans la grande agglomération de Tamarine, la capitale. C'est la raison pour quoi il y a plusieurs modalités de manifestation de troubles à l'ordre public.

Une des modalités a pour cadre le milieu rural, chez les Sessene. ${ }^{8}$ Dans cette entité ethnique, les griots ne sont pas enterrés comme les autres. Ils sont placés dans des baobabs réservés à cette fin. Le but est alors de ne pas souiller la terre. Evidemment, en transgressant les règles élémentaires sur l'inhumation, "ces étranges aberrations" (35), soulèvent un problème sérieux qui ne passe pas inaperçu aux yeux des autorités administratives en charge de l'ordre, sans compter les problèmes d'hygiène publique que cela pose. Les événements de Thaïf ${ }^{9}$ et l'arrestation de Sarkis Biakono ${ }^{10}$ participent également de cette logique de désordre. II reste évident que tous ces événements nécessitent l'intervention des forces de l'ordre. Ils définissent ainsi un cadre général de l'action marqué par l'adversité, le jeu de rapports de force entre la légalité et la volonté des sociétés intermédiaires. Toutefois, tant que ces manifestations sont circonscrites dans un espace géographique périphérique, ils ne constituent pas une menace vraiment sérieuse et, en aucun cas, ne peuvent représenter un danger réel sur la stabilité politique, institutionnelle et sociale du jeune Etat. En bref, ces dérèglements momentanés s'inscrivent dans la vie normale. Bien évidemment, ceci n'est pas toujours le cas.

Le cadre urbain et la qualité des acteurs impliqués donnent une tournure plus importante à certains faits. C'est ainsi que les manifestations orchestrées à la Place de l'indépendance se situent à un autre niveau. Leur ampleur ainsi que les risques de débordement qui s'y rattachent leur donnent une autre allure, parfois dramatique, voire grave, car ces rassemblements peuvent, à bien des égards, secouer le socle du contrat de société. Les manifestations de la colère de "petits," "le déferlement de l'Intersyndicale ${ }^{12}$ de même que la mise à sac du palais présidentiel (257) entrent dans ce cadre.

Toutes ces modalités de trouble à l'ordre public se font jour dans le roman de Cheikh Hamidou Kane. De forme et d'envergure différentes, ces désordres intimement liés à la vie sociale au sein de l'Etat appellent une canalisation, un endiguement, une régulation et un contrôle stricts de la part des autorités investis des pouvoirs légaux en matière de paix et de 
tranquillité publiques. Cette intervention passe par le biais des forces de l'ordre qui sont le bras armé de l'Etat.

\section{II - Les forces de l'ordre}

En matière d'ordre, le précepte wébérien selon lequel seul l'Etat dispose du monopole légal de la violence est de mise dans Les gardiens du temple, tout en donnant l'occasion de faire le portrait, le recensement et d'établir les différentes perceptions sur les forces à l'œ uvre dans le fiction romanesque de Cheikh Hamidou Kane. Chez ce dernier, comme dans la réalité, il y a deux principes qui guident la répartition des tâches: la territorialité et la rationalité. Sur la base de ces deux principes, l'auteur fait apparaître des forces différentes, concurrentes à l'occasion, mais toujours complémentaires au regard de la finalité unique de leur mission.

A la gendarmerie reviennent les zones rurales et l'intérieur du pays. Les unités, communément appelées brigades, avec un effectif peu important, contrôlent une circonscription, généralement calquée sur le découpage administratif. La gendarmerie travaille, entre autres, en étroite collaboration avec les autorités administratives, en tant que pourvoyeuse de renseignements, mais également comme force de coercition à l'occasion. C'est ainsi que dans la région de Kôlé, leur présence se veut d'abord discrète et toujours efficace. Etant au service de la loi, cette présence est dissuasive dans la mesure où elle permet de maintenir l'ordre en endiguant les velléités de trouble à l'ordre public. Néanmoins, les forces de gendarmerie n'hésitent pas à recourir à la manière forte afin d'obliger les citoyens égarés à entrer dans la ligne de la légalité. C'est ainsi qu'à la suite de la réunion tenue à l'initiative de Momar, au sujet "des mesures de politique domaniale appliquées dans la préfecture de Thially" (132), l'intervention des gendarmes ne se fit point attendre. A la limite, celle-ci frise même une certaine forme d'instrumentalisation par le pouvoir, à travers les instructions orientées de ses représentants. Le traitement affligeant et humiliant infligé aux membres des deux groupes antagonistes ressemble à un abus d'autorité, heureusement bien sanctionné par l'application des mesures disciplinaires à l'encontre des auteurs de ces manquements professionnels graves $(134,135)$.

Cette force n'est pas uniquement cantonnée en milieu rural. Elle intervient aussi en milieu urbain, et même jusqu'au cœ ur du pouvoir, en se faisant l'instrument intercesseur des rivalités au sommet de l'Etat. En l'occurrence, c'est elle qui mit fin à la séance parlementaire des conseillers territoriaux, avec l'arrestation de certaines personnalités politiques de 
premier plan (137). ${ }^{13}$ Par son caractère, cette intervention, sujette à controverse par rapport à la légalité, et située au cœ ur de la grave crise politique, fait apparaître, par certains de ses côtés, une certaine forme d'instrumentalisation jamais absente des hautes sphères politiques. Il n'empêche que la gendarmerie jouit d'un crédit très favorable, comme en attestent les jugements positifs des autorités militaires comme civiles: "La gendarmerie a plus de cohésion en son sein et elle obéit à ses chefs" (198).${ }^{14}$ Ainsi se dessine de façon nette le contraste avec la force jumelle qu'est la police.

La police est présente au cœur des agglomérations, suivant la répartition géographique des compétences, dans un système de dualité policière. Elle se noie dans la masse et, en conséquence, son absence d'autorité s'en fait ressentir. Les sergents de villes sont immergés dans les manifestations et ne semblent pas avoir l'ascendant nécessaire pour maitriser le désordre. A la limite, il y a comme une sorte de connivence entre les sergents de ville et les manifestants, les premiers faisant preuve de laxisme profitant à la familiarité recherchée par les seconds: "Les sergents de ville ressemblent aux 'petits,' au-dehors et au-dedans, par la peau et par la tête, par l'estomac" (144). Leurs chefs sont absents et distants ou invisibles. Les policiers sont presque livrés à eux-mêmes. On ne sent pas leur sens de l'organisation. La police paraît divisée: la base se ralliant aux manifestants qu'elle doit contenir, et le sommet de la hiérarchie du côté des autorités, selon l'opinion des subordonnés. La seule fois où le chef apparait, le commissaire Damala, c'est pour donner l'ordre de repli, abandonnant ainsi la place publique aux manifestants (148-50). C'est la raison pour laquelle, contrairement aux hommes du commandant Borko, chef de la gendarmerie, la police ne jouit d'aucune confiance, car son efficacité est douteuse. En tous cas, dans le milieu militaire, et même civil, elle n'a pas bonne presse. L'impression qu'en donne le colonel Dimbo décrit bien la police, au moment où le désordre menace les institutions:

... elle doit être sur les lieux, comme toujours, à agiter ses petits bâtons blancs. Je crois que le cabinet doute de son efficacité, dans l'hypothèse où les choses deviendraient plus graves. Pour ma part, je n'ai pas beaucoup de confiance en elle non plus. Ces braves agents sont très vite débordés. Ils ne sont pas entraînés, ils sont trop mêlés à la population civile, ils... . (217-18)

L'image biaisée que donne la police d'elle-même constitue une entorse au principe de la territorialité. Si les policiers ne sont pas en mesure de tenir leur secteur, eu égard à son importance démographique et stratégique, c'est plus par leur inefficacité légendaire que par le seul facteur numérique. 
Le second principe de compétence, la rationalité, a prévu des défaillances ainsi que des procédés de compensation à ces dysfonctionnements. $\mathrm{Au}$ nombre des ces possibilités, celle de l'intervention des unités mobiles de la gendarmerie, mais aussi et surtout celle de l'Armée.

La situation d'urgence décrétée par les autorités est le cas de figure, par excellence, qui autorise l'intervention de l'armée (176). Elle n'est pas une force de même nature que les deux précédentes. L'armée n'a pas les mêmes équipements, ni le même entraînement que la gendarmerie et la police, pour faire face aux manifestants. Son intervention est toujours délicate, voire problématique, car elle peut avoir des résultats inattendus du fait de l'impact dramatique qu'elle pourrait revêtir. Le général Moriko, patron de cette force, le sait, et maintient une distance réelle vis-à-vis des autorités, afin d'éviter toute possibilité d'instrumentalisation. En effet, il se base sur des fondements légaux qui encadrent de façon formelle l'intervention de l'armée, et prend une hauteur suffisante par rapport aux événements en cours, sans entrer dans une logique d'opportunité pouvant déboucher sur la prise du pouvoir. C'est pourquoi cette force suit de près le cours de choses et n'intervient que de façon très symbolique, par l'acquisition de renseignements et par l'envoi d'un petit détachement commandé par le sergent Malamine. Enfin, son intervention pourrait être appuyée par les forces de l'armée de la colline. ${ }^{15}$

Ainsi donc, ces trois forces principales se distinguent sur beaucoup de plans. La police et la gendarmerie, notamment, n'ont pas les mêmes images aux yeux de Cheikh Hamidou Kane. Peut-être que la différence tient au fait de la militarité de la gendarmerie qui la rapproche plus de l'armée et de ses caractéristiques. Mais l'image des forces, c'est surtout celle de ses figures marquantes.

\section{III - Les figures marquantes des forces de l'ordre}

Chez Cheikh Hamidou Kane, l'iconographie des forces de l'ordre c'est aussi l'image des personnes physiques individuelles ou collectives très représentatives de ces forces, du fait de leurs relations avec le peuple, dans la rue, ou avec les autorités politiques. Après un choix, apparaissent, à nos yeux, trois grandes figures principales qui marquent Les gardiens du temple, eu égard à leur rôle prépondérant et surtout à leur empreinte dans la séquence des événements. Il s'agit du sergent Malamine, de l'unité de gendarmerie mobile présente à la Place de l'Indépendance et de la figure emblématique du général Moriko. Toutes ces personnes se distinguent pour donner un cachet particulier à l'image globale des forces de l'ordre. 
Le sergent Malamine de l'unité parachutiste est le premier cliché complet que l'auteur donne de l'armée nationale. Malamine résume à lui tout seul l'image générique du militaire. Avec ses muscles et son physique imposant, il est la caricature du militaire en trois dimensions. De surcroît, le sergent Malamine adopte un comportement et une attitude typiques du militaire, rayonnant aussi bien sur ses hommes que sur la population: "Mitraillette au poing, visière aux yeux, ceinturon bouclé, taille guêpe, poitrail avantageux et allure de fauve, le sergent Malamine monte et descend l'avenue du Peuple... . Il marche, et le battement de ses semelles sur l'asphalte irradie dans tout son corps des secousses rythmiques. La meute immobile de ses hommes le contemple" (205).

La légende qui auréole sa personnalité contribue également à faire de ce militaire un symbole et une fierté. ${ }^{16}$ Tout en se positionnant devant le palais de la république et en face des manifestants, il n'inspire pas crainte et répulsion. Le sergent Malamine est un patrimoine qui appartient au peuple, car les jeunes s'identifient à lui et le lui montrent à travers leurs acclamations (210). Par conséquent, les manifestants font communion avec lui tout en respectant la distance qui doit les séparer. De son côté, le sergent Malamine n'est pas indifférent à ce culte qu'on lui voue. I1 le ressent dans son fors intérieur et bonifie par là-même son ascendant sur ses hommes par ce surplus de légitimité populaire. C'est pourtant sur lui que tombe le moment du paroxysme de la manifestation, avec la mise en sac du palais, mais le sergent s'en sortit sans écorcher et son image et celle de l'armée (257).

A côté de ce visage éminemment humain, pour une force militaire destinée en priorité à la guerre, il y a, a contrario, celui de l'escadron de gendarmerie mobile. A la différence de Malamine, l'escadron est un être collectif. Il n'a ni sentiment, ni état d'âme. C'est une personnalité distincte dont la mesure se prend uniquement à la lumière de son efficacité sur le terrain, dans le contexte singulier du maintien ou de rétablissement de l'ordre. Face à des manifestants déterminés, car n'ayant "plus de choix qu'entre la victoire et la répression" (167), c'est l'unité spécialisée qui prend en compte les travailleurs qui manifestent. Et contrairement aux sergents de ville immergés dans la foule, les gendarmes font bloc et prennent de la distance. Ils sont bien équipés en matériel adéquat et prêts à agir efficacement, c'est-à-dire vigoureusement. Cheikh Hamidou Kane les présente sous un jour déshumanisé, fait de dépersonnalisation et de rupture physique et sentimentale vis-à-vis de la population. D'ailleurs, il apparaît bien que le gendarme n'existe pas individuellement dans le cadre du maintien de l'ordre. Seule l'unité a une existence et une capacité 
physique efficiente. C'est l'unité élémentaire, dans sa cohésion massive et impressionnante qui supplante les individualités et devient l'entité insécable:

Ils ont des habits kaki. Ils sont couverts de fer et de cuir: ils en ont dans leurs casques, dans leurs armes, dans leurs souliers, leurs véhicules, leurs boucliers. Ils sont forts et nourris de viande par le régime dont ils sont les enfants choyés. On ne voit pas leurs yeux. Il se déplacent en masses compactes et hérissées de fusils. Ils ont le droit de frapper. Ils sont protéges par le cuir, le fer et la loi. Ils sont puissants et sacrés, comme des divinités secondaires (181).

Ainsi, en fondant les gendarmes pris individuellement dans l'anonymat collectif de l'escadron, Cheikh Hamidou Kane crée un être nouveau, un deus ex machina dont la seule finalité est l'application de la loi, parfois au grand dam de la foule. La mort du jeune Sambatio en est la parfaite illustration, suite à la charge de l'escadron de gendarmerie (18788).

En clair, l'escadron symbolise la force au service de l'Etat et de la loi, lesquels se caractérisent par leur impersonnalité froide. Sa raison d'être justifie l'emploi de tous les moyens mis à sa disposition. L'escadron n'est pas une individualité responsable, comme peut l'être le général Moriko.

En tant que chef militaire et plus haut responsable de l'armée, le général Moriko est l'exemple accompli du militaire, avec cette nouveauté qu'il n'incarne ni le tyran, ni l'opportuniste, encore moins des penchants pouvoiristes. Cette figure marquante des forces de l'ordre se spécifie, entre autres, par son désintéressement, son sens manœ uvrier, alliant tact et souplesse, et par son souci de l'intérêt général, toutes qualités qui peuvent forcer l'admiration et consacrer une certaine forme de noblesse militaire.

Le désintéressement du général Moriko atteint son paroxysme avec l'expression de son idée de se démettre des fonctions à sa charge. En effet, suite au désordre grave induit par le déferlement de l'Intersyndicale, puis la mise à sac du palais et l'exfiltration du président, le pouvoir politique s'est retrouvé dans la rue, entre les mains de personne. Alors, en tant que dépositaire de la force armée, le général Moriko aurait pu s'emparer du pouvoir, en profitant du désordre généralisé et en arguant une volonté de restaurer la paix et la tranquillité publique: "Je ne veux pas le pouvoir et, si je le voulais, je n'aurai eu qu'à me baisser pour le ramasser" (279). Au contraire de tout cela, il s'est retrouvé dans la situation de devoir mettre en jeu et ses intérêts personnels et sa carrière militaire, en faisant preuve de probité et de détachement. ${ }^{17}$ 
La seconde caractéristique du général Moriko est son sens manœ uvrier. Par ses manières de faire, il a su épargner le peuple d'un bain de sang inutile, qui aurait l'inconvénient majeur d'aggraver la situation conflictuelle déjà à son comble. Pour lui, la tactique a consisté à refuser de façon souple et prudente l'offre d'intervention de l'armée de la communauté ${ }^{18}$, et surtout à s'interdire toute implication de l'armée devant aboutir logiquement à l'ouverture du feu sur les manifestants. Ainsi, pendant toute la durée de la crise, le général Moriko a tenu l'armée à l'écart des événements ${ }^{19}$, en exerçant un commandement rigoureux ne faisant dériver les ordres que de lui seul $(219,222)$. Son sens manœ uvrier s'est même étendu à sa tentative réussie d'apaisement de la situation par une adresse à la foule de manifestants.

C'est moi. Moriko. Mes compatriotes, il faut nous garder de la honte. Si vous pouvez être tristes, si vous pouvez désirer la justice, si vous avez faim et si vous voulez la dignité, aussi ne répandez le sang de personne. Ici, aujourd'hui, que la violence soit le privilège de l'armée nationale seule. Je l'utiliserai dans la justice jusqu'à tant que la honte soit conjurée. Je viens de l'utiliser dans l'honneur, pour maintenir la justice. Aidez-moi! Rentrez dans vos foyers! (258)

En faisant ainsi appel à des valeurs partagées, il sauve la situation, en fin tacticien et stratège accompli.

Enfin, le général Moriko est la personnification de l'intérêt général. Non content de sauver la situation et d'épargner la collectivité de sombrer dans une violence regrettable, c'est le général lui-même qui organise la pré-conférence nationale entre le peuple et le pouvoir. En convoquant dans une enceinte militaire l'échantillon représentatif du peuple ${ }^{20}$ en face de Jérémie Laskol, le président au pouvoir presque perdu, le général Moriko sauve une seconde fois les meubles en réalisant la prouesse politique qui consiste à réconcilier le peuple avec lui-même, et avec les hommes politiques, après une période de troubles graves. C'est au cours de cette longue nuit de réunion qu'il s'est agi “de déterminer l'ensemble des sujets à débattre et d'établir la liste la plus représentative possible de tous les protagonistes qui devraient prendre part à cette concertation" (3I0). La figure emblématique du général Moriko est celle du militaire dans toute la grandeur de la fonction et dans toute la noblesse de son être pour la défense de l'Etat.

L'iconographie des forces de l'ordre fait ressortir l'image d'une armée loyale. Celle-ci s'est refusée de rentrer dans la spirale de la violence et des coups d'état dans une Afrique cherchant ses repères, à l'aube des indépendances. Face à un pouvoir dans la rue, et également face aux 
injonctions poussant à la répression aveugle, Cheikh Hamidou Kane fait le choix de la description d'une force républicaine se refusant à toute forme d'instrumentalisation par le pouvoir, et à toute libération d'appétits opportunistes d'accaparement du pouvoir. Malgré les défauts constatés par-ci par-là, ainsi que les abus inévitables en matière de police administrative, l'auteur dresse donc, dans une réalité massive et dans une construction romanesque, le portrait fort rare d'une armée loyale dans cette Afrique des années 60 , une force cantonnée à ses droits et devoirs. Ainsi, par le biais de la fiction, Les gardiens du temple est une $œ$ uvre d'exception.

Le refus de l'instrumentalisation s'étend à la puissance coloniale qui, par ses forces stationnées dans le pays et au regard de ses relations particulières avec le jeune Etat, a tout fait pour s'immiscer dans et influencer par infléchissement le cours des événements.

Enfin, malgré l'omniprésence de la question cruciale de l'ordre au regard des libertés collectives et publiques, cette œ uvre qui est apparue bien des lustres après l'ouvrage inaugural, $L$ 'aventure ambiguë, recèle des passerelles aussi intéressantes pour faire la jonction thématique et procédurière entre les deux textes de Cheikh Hamidou Kane. ${ }^{21}$

\section{Université Cheikh Anta Diop de Dakar}

\section{Notes}

1. Voir Diouf, Les formes du roman négro-africain de langue française.

2. Barthe, Le degré zéro de l'écriture 11-24.

3. Le roman de Cheikh Hamidou Kane pourrait s'intégrer parfaitement dans le cadre de la représentation sans complaisance des mœ urs politiques africaines de l'indépendance, au même titre que Le Cercle des tropiques, Les Soleils des indépendances, et d'autres romans.

4. Mauriac, Le romancier et ses personnages.

5. Chevrier, "L'image du pouvoir..." 8.

6. Cheikh Hamidou Kane, Les gardiens du temple. Les pages indiquées entre parenthèses vont se référer à ce texte.

7. Les gardiens page 151 et suivantes.

8. Les gardiens page 31 et suivantes.

9. Les gardiens page 135 et suivantes.

10. Les gardiens page 152 et suivantes. 
11. Les gardiens page 139 et suivantes

12. Les gardiens page 165 et suivantes.

13. Tarman Dankaro, le vice-président.

14. Voir aussi Les gardiens 217.

15. Voir les agissements et procédés de Malterre ayant pour finalité le destitution du général Moriko, au profit du colonel Dimbo, page 240 et suivantes et page 277 , ainsi que la visite suggestive du général Roblot, page 229 et suivantes.

16. Les gardiens page 205 et suivantes.

17. Les gardiens page 269 et suivantes.

18. Les gardiens 234 et page 294 et suivantes.

19. Les gardiens page $222,239,287$ et 288 .

20. Matara l'apprenti chauffeur, Delmo le syndicaliste et professeur de mathématiques, Malam Sango le maître d'école coranique Tchalao le prêtre, Manira le commerçant, Daba Mbaye l'historienne et Djarom le paysan.

21. Cet article commencé en 2001 doit en grande partie sa conclusion à ma rencontre avec Monsieur Cheikh Hamidou Kane, le 18 novembre 2003 à Libreville (Gabon), en marge de la Conférence internationale organisée par l'UNESCO sur Le dialogue interculturel et la culture de la paix en Afrique et dans la région de Grands lacs (18-20/11/2003).

\section{Ouvrages Cités}

Barthes, Roland. Le degré zéro de l'écriture. Paris: Seuil, 1953.

Chevrier, Jacques. "L'image du pouvoir dans le roman africain contemporain." L'Afrique littéraire 85.4 (1989).

Diouf, Madior. Les formes du roman négro-africain de langue française 1920-1976. Thèse de Doctorat d'Etat de Lettres Modernes. Sous la direction de Mr le Pr. Mohamadou Kane. Dakar: Université Cheikh Anta Diop (UCAD), 1990-1991.

Cheikh Hamidou Kane. Les gardiens du temple. Abidjan: NEI, 1996. - L'aventure ambiguë, Paris, Juliard, 1961.

Mauriac, François. Le romancier et ses personnages. Paris: Corréa, 1933. 